\title{
Hereditary Sensory Autonomic Neuropathy Type IV in a Newborn
}

\author{
Dr. Sudipta Kar ${ }^{1}$, Dr. Chinmoy Lath ${ }^{1}$, Dr. Manik Mondal ${ }^{1}$, \\ Dr. Angshumitra Bandyopadhyay ${ }^{1}$, Dr. Rajarshi Basu ${ }^{1}$, Dr. Biswajit Mondal ${ }^{1}$ \\ ${ }^{\text {I}}$ (Department of Pediatric Medicine, NRS Medical college/ The West Bengal University of Health Sciences, \\ India)
}

\begin{abstract}
Hereditary sensory autonomic neuropathy Type IV is an autosomal recessive disorder due to lack of maturation of small myelinated and unmyelinated fibers of peripheral nerves, which convey sensation of pain and temperature. There is anhidrosis due to lack of innervation of normal sweat glands resulting in recurrent episodes of hyperpyrexia. These patients usually present in late infancy. Here we present a case of HSAN Type IV who presented with fever from day 2 of life and so the possibility of HSAN should be kept in mind while dealing a case of fever without focus in a neonate.
\end{abstract}

Keywords: Anhidrosis, Hereditary sensory autonomic neuropathy, Hyperpyrexia, Insensitivity to pain, Neonate.

\section{INTRODUCTION}

Hereditary sensory and autonomic neuropathy (HSAN) is a rare syndrome characterized by congenital insensitivity to pain, temperature changes and by autonomic nerve formation disorders. HSAN is classified into five types: sensory radicular neuropathy (HSAN I), congenital sensory neuropathy (HSAN II), familial dysautonomia or Riley Day Syndrome (HSAN III), congenital insensitivity to pain with anhidrosis (HSAN IV) and congenital indifference to pain (HSAN V)[1]. HSAN type IV inherited as an autosomal recessive trait, presents usually during late infancy and is characterized by recurrent episodes of unexplained fever, failure to thrive, absence or decreased perspiration (despite normal sweat glands), insensitivity to pain, mental retardation and self mutilation [2]. Only few case reports of HSAN are available from India [3-8]. We present here a newborn presenting with features suggestive of HSAN Type IV which is not frequently reported in this age group.

\section{HEADINGS}

1.Case history: A 5 day old baby boy presented with repeated episodes of high grade fever since Day 2 of life. Despite high grade fever baby was sucking well, passing urine adequately and there was no associated complain of respiratory distress, convulsion, inconsolable cry or lethargy.

2.Examination: Baby was alert and playful, Heart rate $160 / \mathrm{min}$, Respiartory rate $52 / \mathrm{min}$, Blood pressure $62 / 46$ $\mathrm{mmHg}$, Temperature varied from $102^{\circ} \mathrm{F}-106^{\circ} \mathrm{F}$. Pallor, cyanosis, clubbing, edema and jaundice was absent. Skin was dry and hot without any rash. Hairs and nails were normal. There was no evidence of any sweat even on meticulous examination. Response to sensory stimulation was preserved but there was absolute no response to pain sensation. Movement of all four limbs was intact with preservation of neonatal and deep tendon reflexes. 3.Investigations: Sepsis screen and CSF study was normal. Culture showed no growth in blood, CSF or urine. Chest x-ray was normal. Skin biopsy showed presence of sweat glands in dermis with retention of pilosebaceous structure [Fig. 1]. Nerve Conduction Velocity (NCV) of upper and lower limbs showed normal Sensory Nerve Action Potential (SNAP) latency, conduction velocity \& amplitude. Pilocarpin iontophoresis test revealed very scanty sweats. Sural nerve biopsy showed reduction in number of myelinated fibres [Fig 2].

4.Treatment :Baby was managed conservatively and a constant vigilance was undertaken to avoid any injury to skin or bones. 


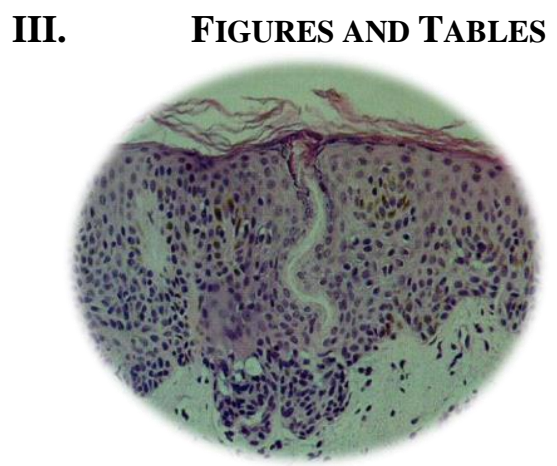

Fig 1: Skin biopsy showing presence of sweat glands in dermis with retention of pilosebaceous structure.

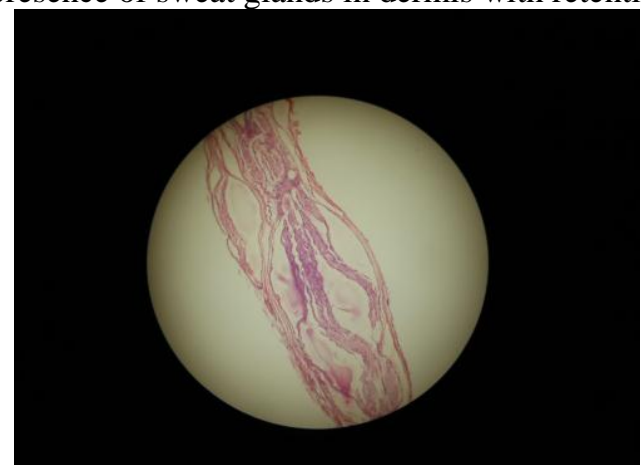

Fig 2: Sural nerve biopsy shows reduction in number of myelinated fibres.

IV.

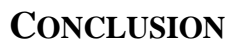

With the above case discussion, it can be concluded that we are dealing with a case of fever without any focus and with insensitivity to pain sensation in a newborn baby. After reliably excluding the common causes of fever in this age group, this case is suggestive of Anhidrotic ectodermal dysplasia or Hereditary Sensory Autonomic Neuropathy. Absence of nail and hair abnormalities and presence of normal sweat glands on skin biopsy exclude the anhidrotic ectodermal dysplasia. The classification of various types of HSAN is based on the inheritance pattern, clinical features, and systems of neurons predominantly affected. HSAN I is autosomal dominantly inherited with symptoms begin in the second decade or later. There is loss of pain and temperature sensation but preservation of tactile sensation. Sural nerve biopsy shows loss of unmyelinated fibres more than myelinated fibres. HSAN II is an autosomal recessive disorder with onset in infancy. There is generalized pansensory loss. Autonomic disturbances included bladder dysfunction, impotence and distal anhidrosis. Motor function is preserved but tendon reflexes are lost. There is loss of myelinated fibres in the sural nerve biopsy. HSAN III is also autosomal recessively inherited affecting mostly Ashkenazi Jews. Nerve biopsy shows reduced number of unmyelinated fibres. HSAN IV is an autosomal recessive disorder associated with bouts of pyrexia, anhidrosis and mental retardation. Nerve biopsy reveals reduced number of small myelinated fibres. HSAN V is an autosomal recessive disorder with onset at birth and normal sweating. Motor functions and tendon reflexes are normal. Sural nerve biopsy shows selective reduction in the number of smaller myelinated fibres. With the above experience gathered, it is suggested that even in a newborn presenting with high grade fever of unknown origin, the entity called HSAN should be kept in mind.

\section{REFERENCES}

[1] Dyck PJ. Neuronal atrophy and degenration predominantly affecting peripheral sensory and autonomic neurons. In: Dick PJ, Thomas PK, Griffin JW, Low PA, Griffin JW, Low PA, et al.eds. Peripheral Neuropath. Philadelphia: WB Saunders. 1993; pp. $1065-1093$

[2] Haworth AE, Ellison DW, Thomas NH, Walker J, Cook LJ. Hereditary sensory and autonomic neuropathy with anhidrosis (type IV). J R Soc Med 1998; 91: 84-86.

[3] Mehta K. Familial dysautonomia in a Hindu boy. Am J Dis Child 1978; 132: 719.

[4] Puri S, Menon PS, Verma A, Swaminathan S, Sarkar C. Hereditary sensory neuropathy: Type II. Indian Pediatr 1990; $27: 744-747$.

[5] Thakur LC, Chandran V, Anand KS. Congenital sensory neuropathy with anhidrosis. Indian Pediatr 1992; $29: 1046-1048$.

[6] Balachandran C, Sabitha L, Kantahraj GR. Hereditary sensory autonomic neuropathy-type II in siblings. Indian J Lepr 1996; 68: 373- 374 .

[7] Krishna Kumar R, Shashi Kiran ND, Subba Reddy VV. Congenital insensitivity to pain (hereditary sensory and autonomic neuropathy). HSAN: a report of two cases. J Indian Soc Pedod Prev Dent 2002; 20: 51-53.

[8] Basu S, Paul DK, Basu S. Four siblings with type II hereditary sensory and autonomic neuropathy. Indian Pediatr 2002; 39: 870874. 